\title{
Adaptive Optimization Method of Resource Structure in Uncertain Environment Based on Cloud Genetic Algorithm
}

\author{
Mengchen Liao ${ }^{1}$, Peng Sun ${ }^{1,2}$, Jieyong Zhang ${ }^{1}$ and Junsheng $\mathrm{Wu}^{3}$ \\ ${ }^{1}$ Information and Navigation College, Air Force Engineering University, Xi'an, China \\ ${ }^{2}$ School of computer science, Northwestern Polytechnical University, Xi'an, China \\ ${ }^{3}$ School of software and microelectronics, Northwestern Polytechnical University, Xi'an, China
}

\begin{abstract}
Aiming at the influence of uncertain factors that impact on tasks and platforms in battlefield environment, adaptive optimization method based on uncertain event is proposed. Taking the uncertainty of platform loss and task implementing time into consideration, established a resource structure optimizing model which takes the minimum mission execution time as object function. And a Resource Structure Based on Cloud Genetic Algorithm is designed to adjust or redesign the platform resource structure according to the influence that uncertain events impact on organization resource capability while triggering optimization. The availability and validity of the algorithm is proved by demonstrating a simulation case of joint campaign.
\end{abstract}

Keywords-platform-task relation; uncertainty; resource structure; adaptive optimization; cloud genetic algorithm

\section{INTRODUCTION}

The mission planning problem of control and command (C2) organization is allocating platform resources to a series of tasks in terms of constraints [1][2][3]. With the battlefield environment become more and more complicated and changeable, uncertain factors affect the allocation relationship between tasks and platforms in most case [4].

Complex and varied mission environment is the main reason of generated uncertainties, it will lead to the change of organizational resource capability, and then affecting the mission to complete [5]. So take the impact of uncertainties on resource requirement and organizational capabilities into consideration will be closer to the actual battlefield. To face up to complex and uncertain mission environment, scholars are proposed the adaptability optimization of mission planning problem [6] [7], which can keep the great performance of the assignment relationship between the platform and the task in view of the changing mission environment. How to improve the timeliness and robustness of organizational capability while guaranteeing the effectiveness is still a problem to be solved.

This paper proposes a resource structure optimization method based on cloud genetic algorithm. The mission execution time is defined as the objective function to optimize the planning Model [8], combined with cloud model theory [9] to optimize the genetic algorithm. This method can make a fast adjustment of the allocation according to the influence of uncertain events on organizational capability, on the other hand, method can shorten the mission completion time. Finally, the applicability and effectiveness of the method are verified by simulation case.

\section{PlanNING PROBLEM DESCRIPTION AND FORMULATION}

\section{A. Problem Feature Definition}

In the process of allocation, the key entities in our planning problem are the following.

Definition 1-Task: A task is the smallest basic unit decomposed by the mission, it is executed by specific physical resources. Task is characterized by the following properties:

1) Start time of task $B T_{i}$;

2) Processing time of task $L T_{i}$;

3) The location of the task $T P_{i}=\left(x_{i}, y_{i}\right)$;

4) Resource requirement vector $T R_{i}=\left\{T R_{i l}\right\}(l=1,2, \ldots, L)$, represent the number of type $\mathrm{l}$ resources required to successfully process a task $T_{i}$.

Definition 2 - Platform: Platform is the entity of organizational resource capacities, it is the physical resources dealing with tasks. Each platform has the following attributes:

1) The initial location of the platform $P P_{k}=\left(x_{k}, y_{k}\right)$

2) The maximum moving speed of the platform $v_{k}$;

3) Resource capability vector $P R_{k}=\left\{P R_{k l}\right\}(l=1,2, \ldots, L)$, where $P R_{k l}$ is the number of resources category $l$ of platform $k$ contain.

Definition 3-Mission Completion Time TFT $\left(T, G_{R S}\right)$ :

$$
\operatorname{TFT}\left(T, G_{R S}\right)=\max \left(B T_{i}+L T_{i}\right)-\min B T_{j}
$$

The total completion time of the mission in the case of an assignment relationship $G_{R S}$ is the interval between the finish time $B T_{i}+L T_{i}$ of the last task $T_{i}$ and the start time $B T_{j}$ of the first task $T_{j}$. 


\section{B. Description of Uncertainty}

Due to the complex battlefield environment, uncertain factors is spoiling the stable relationship between task and platform, and further affecting the resource capacities of the C2 organization. We define those kinds of uncertainties as uncertain events, they are constituted by platform loss events and task processing time change events.

Definition 7-Platform Loss Events: The platform loss event indicates the ability of the platform resources is reduced in terms of the uncertainty elements on the battlefield during the mission. Platform loss event attribute are denoted as $\left(e_{j}, E R_{l}^{j}, A P_{P U}\right)$ :

Where $e_{j}$ denotes the serial number of loss event, $E R_{l}^{j}$ is the number of resource type $l$ lost by platform $P_{j}$ under this event, $A P_{P U}$ indicates the occurrence probability of this event.

Definition 8-Task processing time change events: Task processing time change event is the unexpected uncertainty lead to an interval between the task actual processing time and expected processing time. Task processing time change event attributes are denoted as $\left(e_{i}, \Delta L T_{e_{i}}^{T U}, A P_{T U}\right)$ :

Where $e_{i}$ denotes the serial number of processing time change event, $\Delta L T_{e^{i}}^{T U}$ denotes processing time Variation of $\operatorname{task} T_{e_{i}}, A P_{T U}$ indicates the occurrence probability of the event.

\section{Modeling Formulation}

The core of resource structure for C2 organization is the relationship of task-platform assignment $R_{P-T}$, the assignment Reflects resource mapping association between tasks and platforms. $R_{P-T}$ design is actually allocate appropriate platform resources to tasks at the right place and the right time.

Configuration of organizational resource capability is decided by the relationship between task and platform, different $R_{P-T}$ leads to different resource capabilities. In process of executing tasks, the occurrence of uncertain events resulting in decrease of organization resource capabilities, the resource structure adaptive optimization is triggered at time $t$. In order to enhance resource capabilities, the way of adjusting the assignment of $R_{P-T}$ to improve capabilities is proposed.

When the resource structure $G_{R S}$ is adjusted at time $t$, adaptive optimization of resource structure is formulated by

$\min \operatorname{TFT}\left(T(t), G_{R S}^{\prime}\right)$

$$
\text { s.t. }\left\{\begin{array}{l}
\sum_{j=1}^{N_{p}} R_{P-T}(i, j)=1, i=1,2, \ldots,|T(t)| \\
R_{P-T}(i, j) \in\{0,1\}, i=1,2, \ldots,|T(t)|, j=1,2, \ldots, N_{p}
\end{array}\right.
$$

Where $T(t)$ is the set of tasks which is affected by the uncertain event at time $t, G_{R S}^{\prime}$ is the new resource structure after adaptive optimization.

\section{AdAPTIVE Optimization Module}

\section{A. Optimize Processes}

In order to overcome the instability of organization structure brought by uncertainty, cloud genetic algorithm for resource structure optimization is proposed. This method is aiming at solving the problem which has initial resource structure, a more stable resource structure is obtained after optimization.

The flowchart of optimization process is shown in FIGURE I.

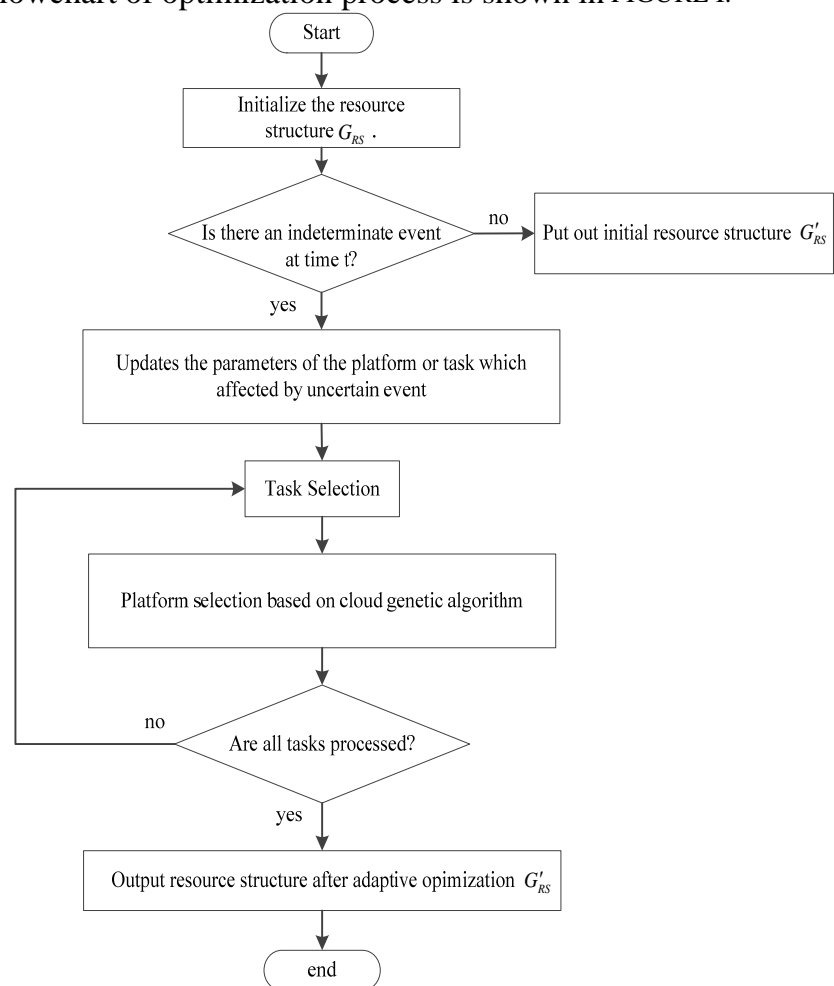

FIGURE I. FLOWCHART OF RESOURCE STRUCTURE BASED ON CLOUD GENETIC ALGORITHM

\section{B. Cloud Genetic Algorithm}

Genetic algorithm will be used to solve NP-hard problem. In order to improve the performance of genetic algorithm, cloud model theory is utilized. Reflecting better randomness and stability tendency by taking advantage of cloud model, meanwhile small population size and strong search ability is also a superiority after improved, Cloud genetic algorithm is selected.

In process of task selection, weighted length algorithm (WL) is used to determine the task priority:

$$
P_{i}=L T_{i}+\max _{j \in \text { out }(i)} P_{j}+\frac{\sum_{j \in \text { out }(i)} P_{j}}{\max _{j \in \text { out }(i)} P_{j}}
$$

Where $L T_{i}$ denotes the processing time of task $T_{i}$, out (i) is the subsequent tasks set of $T_{i}$. Higher priority of task means 
earlier executed than lower.

\section{1) Coding scheme}

As for current executing task $T_{i}$, the available platforms are selected from the set of free platforms $P_{\text {free }}$. In this paper, dual-string binary code are used to represent the allocation of the task of the platform. The upstream string indicates the serial number of the platform in $P_{\text {free }}$, and the downstream string uses 0 or 1 to indicate the selected status of the platform.

TABLE I. ENCODING STRING STRUCTURE

\begin{tabular}{|c|c|c|c|c|}
\hline $\begin{array}{c}\text { order } \\
\text { names }\end{array}$ & \multicolumn{4}{|c|}{ value } \\
\hline $\begin{array}{c}\text { serial } \\
\text { number }\end{array}$ & $S(1)$ & $S(2)$ & $\ldots \ldots$ & $S($ free $)$ \\
\hline $0-1$ value & $G_{S(1)}$ & $G_{S(2)}$ & $\ldots \ldots$ & $G_{S(\text { free })}$ \\
\hline
\end{tabular}

2) Fitness function

Within the solution space, each candidate solution corresponds to a different task platform assignment, and the fitness value of the solutions represent the performance of different allocation. Meanwhile, the fitness value should reflect the performance of maximizing the efficiency of task execution. Therefore, time priority coefficient and platform capacity priority coefficient need to be considered in the fitness function.

\section{a) Time priority coefficient $C T\left(P_{u s e}^{(i)}\right)$}

The optimal individuals in the population are the individuals with the smallest task start time, The normalized processing time priority coefficient as follows:

$$
C T\left(P_{u s e}^{(i)}\right)=\frac{S t\left(P_{u s e}^{(i)}\right)-S t_{\min }}{\sum_{n=1}^{p}\left(S t\left(P_{u s e}^{(n)}\right)-S t_{\min }\right)}
$$

Where $\operatorname{St}\left(P_{u s e}^{(i)}\right)$ is the start time of task $T_{i}$ :

$$
\operatorname{St}\left(P_{u s e}^{(i)}\right)=\left(B T_{j}^{(k)}+L T_{j}^{(k)}+\frac{d_{i j}}{v_{k}}\right)
$$

b) Resource capacity priority coefficient $C P\left(P_{u s e}^{(i)}\right)$

Resource capacity redundancy is a important index in allocating platforms to tasks, it is required as small as possible to reduce the current task of the selected platform for the impact of subsquent tasks as follows:

$$
C P\left(P_{\text {use }}^{(i)}\right)=\frac{R_{\text {redund }}\left(P_{\text {use }}^{(i)}\right)+R_{\text {other }}\left(P_{\text {use }}^{(i)}\right)-S R_{\min }}{\sum_{k=1}^{p}\left(R_{\text {redund }}\left(P_{\text {use }}^{(k)}\right)+R_{\text {other }}\left(P_{\text {use }}^{(k)}\right)-S R_{\min }\right)}
$$

Where $R_{\text {redund }}$ is resource redundancy of platform $P_{u s e}^{(i)}$ for task $T_{i}$

$$
R_{\text {redund }}\left(P_{u s e}^{(i)}\right)=\sum_{l=1}^{L}\left(\sum_{m \in P_{\text {use }}^{(i)}} P R_{m l}-R_{i l}\right)
$$

We compute the average resources satisfaction of the selected platform $P_{\text {use }}^{(i)}$ for other tasks in the set of tasks:

$$
R_{\text {other }}\left(P_{\text {use }}^{(i)}\right)=\frac{1}{n u m} \sum_{m \in P_{\text {use }}^{(i)}} \sum_{j \in T_{\text {ready }}} \sum_{T_{i}}^{L}\left(P R_{m l}, R_{j l}\right)
$$

In terms of (6) (7), the best individual of the population as follows:

$$
S R_{\min }=\min _{i \in[1, p]}\left(R_{\text {redund }}\left(P_{\text {use }}^{(i)}\right)+R_{\text {other }}\left(P_{\text {use }}^{(i)}\right)\right)
$$

\section{3) Crossover and mutation operator based on cloud model}

Set the probability of crossover in genetic algorithm as $P_{c}$ and the mutation probability as $P_{m}$. In the later of the algorithm, $P_{c}, P_{m}$ value is set to be small, less of individuals are involved in the variation, and the global search ability is strongest while the optimal individuals are kept.

According to the $\mathrm{X}$-conditional cloud generator, the adaptive adjustment of the crossover and mutation probability in the algorithm as follows:

a) Adaptive crossover probability $P_{c}$ :

$$
\begin{aligned}
& E x=\bar{f} \\
& E n=\frac{f \max -\bar{f}}{C_{1}} \\
& H e=\frac{E n}{C_{2}} \\
& E n^{\prime}=\operatorname{randn}(E n, H e) \\
& P C=\left\{\begin{array}{l}
k_{1} e^{\frac{-\left(f^{\prime}-E x\right)^{2}}{2(E n)^{2}}}, f^{\prime} \geq \bar{f} \\
k_{3}, f^{\prime}<\bar{f}
\end{array}\right.
\end{aligned}
$$

b) Adaptive mutation probability $P_{m}$ : 


$$
\begin{aligned}
& E x=f \\
& E n=\frac{f \max -f}{C_{3}} \\
& H e=\frac{E n}{C_{4}} \\
& E n^{\prime}=\operatorname{randn}(E n, H e) \\
& P m=\left\{\begin{array}{l}
k_{2} e^{\frac{-(f-E x)^{2}}{2(E n)^{2}}} \\
k_{4}, f^{\prime}<\bar{f}
\end{array}, f^{\prime} \geq \bar{f}\right. \\
& C_{1} \sim C_{4}, k_{1} \sim k_{4} \in[0,1]
\end{aligned}
$$

Here, $\bar{f}$ denotes the current mean fitness of the population, $f_{\max }$ is the maximum fitness value of the outstanding individuals in the population, $f$ 'denotes bigger fitness value in the cross operation, and $f$ is the fitness value of the mutation individual in the population. $C_{1} \sim C_{4}$ is control coefficient, $k_{1}, k_{3}$ denotes the cross-parameter, $k_{2}$, $k_{4}$ denotes the variation parameter, where $k_{1} \sim k_{4} \in[0,1]$.

\section{EXPERIMENT RESULTS}

\section{A. Mission Scenario}

The landing campaign of a joint operation is setting for simulation analysis, the timing relation graph of the mission and detailed parameters of the platform and task are described in [10].

\section{1) Uncertain events parameters setting}

a) Platform loss events: Assuming that the probability of platform loss events $A P_{P U}$ obeys the Poisson distribution, which is $A P_{P U} \sim \pi\left(\lambda_{j}\right)$, where $\lambda_{j}$ is probability of the loss event. The serial number $e_{j}$ of platform and resource type $l$ is randomly generated during the simulation.

In the simulation experiment, assumed platform loss events is $E_{P U}=\left\{E_{1}^{p}, E_{2}^{p}\right\}$, and $E_{1}^{p}$ take place in time period [77, 78], the number of resource $l_{6}$ that platform $P_{7}$ loss is $E R_{6}^{7}=1.5$, the mean probability of $E_{1}^{p}$ is $\lambda_{1}=0.03 ; E_{2}^{p}$ take place in time period $[90,91]$ the number of resource $l_{8}$ that platform $P_{16}$ loss is $E R_{8}^{16}=1$, the mean probability of $E_{1}^{p}$ is $\lambda_{2}=0.02$.

b) Task executing time change events: Assuming that the probability of platform loss events $A P_{T U}$ obeys the Poisson distribution, which is $A P_{T U} \sim \pi\left(\lambda_{i}\right)$, The task processing time variation $\Delta L T_{e_{i}}^{T U}$ obeys the uniform distribution, which is $\Delta L T_{e_{i}}^{T U} \sim U\left(t_{\text {lower }}, t_{\text {upper }}\right)$.

In the simulation experiment, assumed task processing time change even $E_{1}^{T}$ take place in time period [52, 53], the processing time of task $T_{12}$ is increased by 2 units, which is $\Delta L T_{12}^{T U}=2$.

\section{2) Algorithm parmeters setting}

In process of adaptive adjustment, population size $p=10$, iterations time $N=100$, initial crossover probability $P_{c}=0.8$, initial mutation probability $P_{m}=0.1$. At the beginning of cloud genetic algorithm, the control coefficient $C_{1}=C_{3}=6 p$, with the generation gradually increasing $(N=50)$, coefficient $C_{2}=C_{4}=15-(N-P / 2)^{2}$.

\section{B. Result Analysis}



FIGURE II. THE INITIAL RESOURCE STRUCTURE AND EXECUTION TIME (TFT=135.15s)



FIGURE III. THE NEW RESOURCE STRUCTURE AFTER OPTIMIZTING AND EXECUTION TIME $(T F T=117.12 \mathrm{~s})$

According to the settings of uncertain event, the event $E_{1}^{T C}$ lengthen the processing time of task $T_{12}$, subsequent of $T_{12}$ may be affected in allocating. The result shows some of tasks which resource requirement is small assigned less platform resource, on the other hand, in order to avoid conflict arising from platform competition, choose others platform which satisfied the requirement of task instead of same platform used by different task.

When $E_{1}^{p}$ is taking place, platform task $T_{2}$ and task $T_{5}$ is affected in terms of platform $P_{7} . T_{2}$ is executing when event happens, the allocation of $T_{2}$ do not change. $T_{5}$ is affected by the impact of uncertain events, platform assignment from the original $P_{5}, P_{7}, P_{19}$ adjust to $P_{3}, P_{5}, P_{19}$ to ensure the implementation of the task, and avoid conflict with the platform selection.

When $E_{2}^{p}$ is taking place, $P_{16}$ used by task T1and task $T_{2}$ is affected. But there is no processing task for this platform in 
time period [90, 91], so the allocation of the relationship between the platform and the task is not changed.

The occurrence of uncertain events triggered the adaptability optimization of the resource structure, and changed the distribution relationship between the platform and the task. The mission completion time was shortened from 135.15 s to $117.12 \mathrm{~s}$, and the resource structure was adjusted to enhance the mission completion effect. The relationship between the tasks and platforms after optimizing is shown in FIGURE III.

\section{SUMMARY}

This paper studies the change of organization resource capacity under the influence of uncertain events, and take the events of platform loss and task processing time changes into consideration, the simulation results show that the method of organizational structure optimization is applicable. There are other many uncertain factors may exist in the mission environment, these factors may influence the decision-making workload and execution ability of organization. Therefore, the problem of complex uncertainties taking into consideration on organizations need to be further studied.

\section{REFERENCE}

[1] Park C, Pattipati K R, and An W. "Quantifying the Impact of Information and Communication Structures via Distributed Auction Algorithm,” 2010 IEEE International Conference on Systems, Man, and Cybernetics. Istanbul, IEEE press, pp. 2200-2207, 2010.

[2] Han X, Bui H, and Mandal Sl. “Optimization-based Decision support software for a team-in-the-loop experiment: Asset package selection and planning," IEEE Trans. on Systems, Man, and Cybernetics-Part A: Systems and Humans, vol.3, pp. 237-251, February 2013.

[3] Sun Y, Yao P Y, Wu J X. "Design method of flattening command and control structure of army organization,” Systems Engineering and Electronics, vol.38, pp.1833-1839, August 2016.

[4] Han X, Bui H, and Mandal S. "Optimization-based Decision support software for a team-in-the-loop experiment: Asset package selection and planning," IEEE Trans. on Systems, Man, and Cybernetics-Part A: Systems and Humans, vol.44, pp. 237-251, March 2013.

[5] Sidoti D, Ayala X, and Han X. "Evaluating the value of information in the presence of high uncertainty,” 18th ICCRTS: C2 in underdeveloped, degraded, and denied operational environments, 2013.

[6] Levchuk G M, Levchuk Y N, and Pattipati K R. "Mapping Flows onto Networks to Optimize Organizational Processes,” 7-th Command and Control Research and Technology Symposium, Monterey,CA,2002.

[7] Levchuk G M, Yu F, and Pattpati K R. "From Hierarchies to Hierarchies Application of Network Optimization," 7-th Command and Control Research and Technology Symposium, 2002,Monterey,CA,2002.

[8] MOU Liang. "Dynamic Adaptive Optimization Methodology of C2 organization Structure under Uncertainty Mission Environment," Graduate School of National University of Defense Technology, 2010.

[9] LI Deyi, and LIU Changyu. "Study on Universality of the Normal Cloud Model,” Engineering Sciences, vol.6, pp.28-34, August 2004.

[10] Levchuk G M ,Levchuk Y N, and Luo J. "Normative design of organizations-part I : Mission Planning,” IEEE Trans. on Systems, Man, and Cybernetics-part A:Systems and Humans, vol.34, pp. 337-350, March, 2004. 